\title{
Spectrophotometry of SN 1987A from the Kuiper Airborne Observatory
}

\author{
By DI A NE H. WOODEN
}

\author{
NASA Ames Research Center, MS 245-6, Moffett Field, CA 94035-1000, USA
}

Explosion calculations of SN 1987A generate pictures of Rayleigh-Taylor fingers of radioactive ${ }^{56} \mathrm{Ni}\left({ }^{56} \mathrm{Ni} \rightarrow{ }^{56} \mathrm{Co} \rightarrow{ }^{56} \mathrm{Fe}\right)$ which are boosted to velocities of several thousand $\mathrm{km} \mathrm{s}^{-1}$. From the KAO observations of the mid-IR iron lines, a picture of the iron in the ejecta emerges which is consistent with the 'frothy iron fingers' having expanded to fill about $50 \%$ of the metal-rich volume of the ejecta $\left(v_{m} \leq 2500 \mathrm{~km} \mathrm{~s}^{-1}\right)$. The ratio of the nickel line intensities $I([N i I] 7.5 \mu \mathrm{m}) / I([N i I I] 6.6 \mu \mathrm{m})$ yields a high ionization fraction of $x_{N i} \geq 0.9$ in the volume associated with the iron-group elements at day 415 , before dust condenses in the ejecta.

From the KAO observations of the dust's thermal emission $(2 \mu m-100 \mu m)$, it is deduced that when the grains condense their infrared radiation is trapped, their apparent opacity is gray, and they have a surface area filling factor of about $50 \%$. The dust emission from SN $1987 \mathrm{~A}$ is featureless: no $9.7 \mu \mathrm{m}$ silicate feature, nor PAH features, nor dust emission features of any kind are seen at any time. The total dust opacity increases with time even though the surface area filling factor and the dust/gas ratio remain constant. This suggests that the dust forms along coherent structures which can maintain their radial line-of-sight opacities, i.e., along fat fingers. The coincidence of the filling factor of the dust and the filling factor of the iron strongly suggests that the dust condenses within the iron, and therefore the dust is iron-rich. It only takes $\sim 10^{-4} M_{\odot}$ of dust for the ejecta to be optically thick out to $\sim 100 \mu \mathrm{m}$; a lower limit of $4 \times 10^{-4} M_{\odot}$ of condensed grains exists in the metal-rich volume, but much more dust could be present.

References to KAO observations of SN 1987A include efforts by principal authors Colgan, Dwek, Erickson, Haas, Harvey, Moseley, Rank, Witteborn, \& Wooden.

\section{Iron in $\mathrm{SN} 1987 \mathrm{~A}$}

In the first week following the explosion of SN 1987A, the rapid radioactive decay of ${ }^{56} \mathrm{Ni} \rightarrow{ }^{56} \mathrm{Co}$ deposited about $40 \%$ of the total ${ }^{56} \mathrm{Ni}$ decay energy into the inner regions of the ejecta forming a hot low density 'nickel bubble' interior to denser overlying layers. This bubble and the denser overlying layers became Rayleigh-Taylor unstable, boosting fingers or bullets of ${ }^{56} \mathrm{Co}$ and iron-group elements to velocities $\sim 3000 \mathrm{~km} \mathrm{~s} \mathrm{~s}^{-1}$, penetrating the outer layers of the ejecta. (Without the 'popping of the nickel bubble' the iron group elements are expected to have reached velocities of only about $1000 \mathrm{~km} \mathrm{~s}^{-1}$.) Models which include this mixing of ${ }^{56} \mathrm{Co}$ into the outer layers of the ejecta are required to fit the bolometric light curve, to account for the early appearance of $\mathrm{x}$-rays and $\gamma-$ rays, and to explain the observed $\gamma$-ray line ratios. These indirect observations which require models with mixing provoke the following questions regarding the emission lines from the iron-group elements: Do the iron lines provide evidence for the finger structures or high velocity bullets? Does the evolution of the lines indicate how much of the ejecta volume is filled by these fingers? Is there evidence for a large variation in density in the bullets or fingers?

The mid-IR iron forbidden lines are ideal for probing the physical conditions of the iron in the ejecta. They are well separated in wavelength, have upper levels within a few thousand degrees of the ground state, have critical densities of about $10^{4} \mathrm{~cm}^{-3}$, and their level populations are well approximated by LTE ( $\mathrm{Li}, \mathrm{McCray}$ \& Sunyaev 1993). The 
strongest mid-IR iron lines include the [Fe II] $a^{6} D_{7 / 2}-a^{6} D_{9 / 2} 25.99 \mu m$ ground-state transition, and the [Fe II] $a^{4} F_{7 / 2}-a^{4} F_{9 / 2} 17.94 \mu m$ and [Fe II] $a^{4} F_{5 / 2}-a^{4} F_{7 / 2} 24.52 \mu m$ excited state transitions. With an excitation temperature of only $554 \mathrm{~K}$, the $25.99 \mu \mathrm{m}$ line intensity is insensitive to electron temperature and most sensitive to local density. The $18 \mu \mathrm{m}$ line with $E_{u} / k=3496 \mathrm{~K}$ and the $24.5 \mu \mathrm{m}$ line with $E_{u} / k=4083 \mathrm{~K}$ depend on electron temperature but have only a fraction of the optical depth as the $26 \mu \mathrm{m}$ line, as shown in Figure 1a. The near-IR [Fe II] $a^{6} D_{9 / 2}-a^{4} D_{7 / 2} 1.26 \mu m$ multiplet member has an excitation temperature greater than $11000 \mathrm{~K}$; the $1.26 \mu \mathrm{m}$ line intensity is very temperature sensitive while its optical depth is negligible.

Figure 1 demonstrates how the electron temperatures derived from the mid-IR line intensity ratios drop substantially when the assumption of optically thin lines (open symbols) is abandoned and the optical depths in the lines are considered (solid symbols and shaded areas). For level populations in LTE, the line intensity is proportional to the product of the upper level population and the escape probability: $I_{u l} \propto N_{u}\left(T_{e}\right) A_{u l} P_{e s c}\left(\tau_{l u}\right)$, where the escape probability is given by $P_{e s c}(\tau)=(1-\exp (-\tau)) / \tau$. The line optical depth is a local phenomenon; in the Sobolev approximation where different velocity components in a line are taken to arise in different physical regions in the ejecta, the optical depth $\tau_{l u}(v)$ in a part of the line depends on the local density in the ejecta at that velocity $v$ (Li et al. 1993 Eq. 9):

$$
\tau_{l u}(v)=\frac{\lambda_{o}^{3} t}{8 \pi} \frac{g_{u}}{G\left(T_{e}\right)} A_{u l} e^{-E_{l} / k T_{e}}\left(1-e^{-\left(E_{u}-E_{l}\right) / k T_{e}}\right) N_{F e^{+}}(v) .
$$

If the line is not velocity-resolved, then the optical depth $\bar{\tau}_{l u}$ represents the average of the local conditions in the nebula. The density drops with time as $N \propto t^{-3}$ as the ejecta expands homologously, correspondingly the line optical depth drops as $\tau \propto t^{-2}$; as the line optical depth drops, the line intensity should increase as $I_{u l} \propto t^{2}$ if the temperature is constant.

As shown in Figure 1b, at 250 days the ratio $I_{18 \mu m} / I_{26 \mu m}$ yields an electron temperature of $T_{e}=6000_{-1000}^{+2000} \mathrm{~K}$ in the optically thin limit (Moseley et al. 1989a). If both ratios $I_{18 \mu \mathrm{m}} / I_{26 \mu \mathrm{m}}$ and $I_{24.5 \mu \mathrm{m}} / I_{26 \mu \mathrm{m}}$ are considered simultaneously, then a solution is reached with $T_{e}=3250 \pm 750 \mathrm{~K}$ and $\bar{\tau}_{26 \mu m}=3 \pm 1$.

At 250 days, only $0.019 M_{\odot}$ of $F e^{+}$are seen in the $26 \mu m$ line (Moseley et al. 1989a), equal to about $1 / 3$ of the total iron mass existing from the radioactive decay of $0.075 M_{\odot}$ of ${ }^{56} \mathrm{Co}$, and consistent with the optical depth of $\bar{\tau}_{26 \mu \mathrm{m}} \simeq 3$ derived from the mid-IR line ratios and a high ionization fraction. The non-detection of the mid-IR [Fe I] and [Fe III] forbidden lines also supports a high singly-ionized iron ionization fraction. To produce the optical depth in the $26 \mu \mathrm{m}$ line, an average local number density of $\mathrm{Fe}^{+}$ions of $\bar{N}_{\mathrm{Fe}+}=2.6_{-1.2}^{+1.6} \times 10^{6} \mathrm{~cm}^{-3}$ is required. This is equal within the uncertainties to the average number density obtained if all the iron is singly-ionized and spread throughout the metal-rich volume (for $x_{F e}=1, M_{F e}(250$ days $)=0.067 M_{\odot}$, and $v_{m}(F W H M)=$ $2500 \mathrm{~km} \mathrm{~s}^{-1}$, then $\bar{N}_{\mathrm{Fe}+}=2.2 \times 10^{6} \mathrm{~cm}^{-3}$ ). If $x_{F e} \simeq 0.6$, as deduced from the optically thin near-IR [Fe II] $1.26 \mu \mathrm{m}$ line at 192 and 225 days (Oliva et al. 1987 and Meikle et al. 1989, respectively), then the iron ions have a volume filling factor of $f=0.5_{-0.2}^{+0.4}$. For an ionization fraction of $x_{F e}=0.8$ and a higher electron temperature of $T_{e} \approx 4000 \mathrm{~K}$, the iron ions have a volume filling factor of $f \geq 0.4$ (as shown by Li et al. 1993 in their simultaneous fitting of many cobalt, iron, and nickel lines). Thus the 'frothy iron fingers' must fill about $50 \%$ of the metal-rich volume in order to produce the observed optical depth of the $26 \mu \mathrm{m}$ line: the iron is seen as the leavening in the $\sim 1 M_{\odot}$ metal-rich volume.

As shown in Figure 1c, at 415 days the ratio $I_{18 \mu m} / I_{26 \mu m}$ yields an electron temper- 



Figure 1. a. $-\tau$ vs $T_{e}$; b\&c.- [Fe II] line intensity ratios $v s T_{e}$ and $\tau_{26 \mu m}$.

ature of $T_{e}=6600_{-1700}^{+2800} \mathrm{~K}$ in the optically thin limit (Haas et al. 1990). The $18 \mu \mathrm{m}$ and the $26 \mu \mathrm{m}$ lines were measured with a velocity resolution of $\sim 400 \mathrm{~km} \mathrm{~s}^{-1}$, thus providing the velocity-resolved intensity ratio $I_{18 \mu m}(\Delta v) / I_{26 \mu m}(\Delta v)$ shown in Figure 2a $((26 \mu m / 18 \mu u) \times$ Fig. 3 from Haas et al. ; private communication from Colgan). The statistically weighted average over the best signal-to-noise points (solid circles in Figure 2a) yields an average velocity-resolved line intensity ratio of $\left\langle I_{18 \mu m}(\Delta v) / I_{26 \mu m}(\Delta v)\right\rangle=$ $2.1 \pm 0.1$. This average velocity-resolved line intensity ratio yields an electron temperature of $T_{e}=4400 \pm 400 \mathrm{~K}$ in the optically thin limit as shown in Figure 1c, equal to that derived by Haas et al. Between 250 days and 410 days the average optical depth in the $26 \mu \mathrm{m}$ line is expected to drop a factor of $\sim 2.7: \bar{\tau}_{26}(410$ days $)=3 \pm 1 \rightarrow$ $\bar{\tau}_{26}(410$ days $)=1.2 \pm 0.4$. Taking this range for $\bar{\tau}_{26}(410$ days $)$, the electron temperature is $T_{e}=3450 \pm 650 \mathrm{~K}$, in the same range as the electron temperature derived at 250 days. 


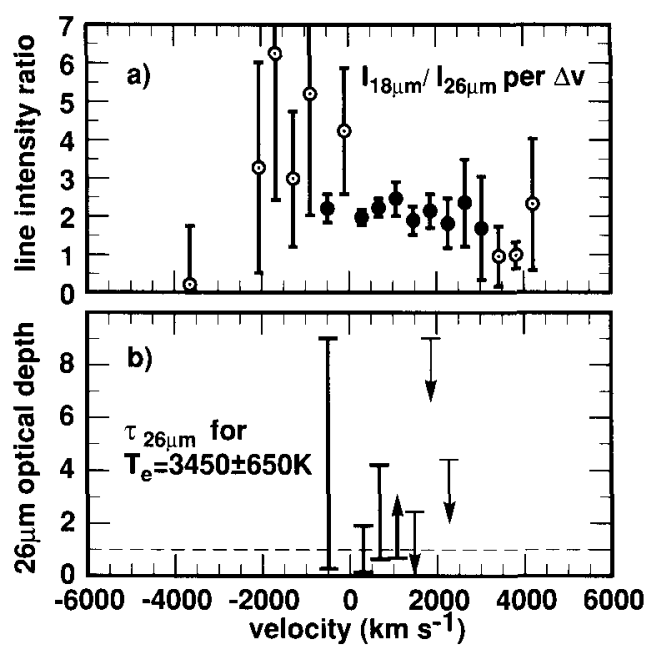

FIGURE 2. (410 days) a.- velocity-resolved line intensity ratio. b. $-\tau_{26 \mu m}$.

Figure $2 \mathrm{~b}$ shows the velocity-resolved optical depth in the $26 \mu \mathrm{m}$ line for $T_{e}=3450 \pm$ $650 \mathrm{~K}$. Different regions of the ejecta corresponding to velocities between $1000 \mathrm{~km} \mathrm{~s}^{-1}$ and $-3000 \mathrm{~km} \mathrm{~s}^{-1}$ have $26 \mu \mathrm{m}$ line optical depths ranging from less than one to several, and are thus consistent with an average optical depth of $\bar{\tau}_{26 \mu m} \simeq 1$.

Moseley's measurement of the $26 \mu \mathrm{m}$ line intensity at 395 days yields an $\mathrm{Fe}^{+}$mass of $0.033 M_{\odot}$ (Dwek 1988), an increase of only 1.7 over the $0.019 M_{\odot}$ deduced at 250 days; the iron mass does increases due to an increase in transparency of the $26 \mu \mathrm{m}$ line, but not by the factor of 2.7 from the expected drop in optical depth. The measurements of the $26 \mu \mathrm{m}$ line by the Cooled Grating Spectrometer at 250 days (Erickson et al. 1988; $\Delta v=1900 \mathrm{~km} \mathrm{~s}^{-1}$ ) and 410 days (Haas et al. 1990; $\Delta v=400 \mathrm{~km} \mathrm{~s}^{-1}$ ) show, however, no increase in the $26 \mu \mathrm{m}$ line intensity between these two epochs. Haas et al. (1990) argue that the failure of the $26 \mu \mathrm{m}$ line strengths to increase between 250 days and 410 days implies that the line emission arises from optically thin iron, and the hidden iron is very optically thick. This argues against the line intensity ratios providing a meaningful average optical depth $\bar{\tau}_{26 \mu m}$, and argues in favor of regions with $\tau_{26 \mu m}<1$ and other regions with $\tau_{26 \mu m} \gg 1$.

If there are regions in the ejecta with $\tau_{26 \mu m} \gg 1$, they probably would not be seen in the $18 \mu \mathrm{m}$ line profile, but could seen in the near-IR $1.26 \mu \mathrm{m}$ line profile $\left(\tau_{1.26 \mu \mathrm{m}}=\right.$ $(0.006-0.009) \times \tau_{26 \mu m}$ for $\left.T_{e}=3000-5000 \mathrm{~K}\right)$. Haas et al. (1990)'s Fig. 3 shows the comparison of the $18 \mu \mathrm{m}$ line profile at 407 days with the $1.26 \mu \mathrm{m}$ line profile at 377 days. The central portions of the near-IR and the mid-IR line profiles are the same. Furthermore, the detailed shapes of the near-IR forbidden line profiles remain essentially unchanged from 250 days through about 600 days (Spyromilio, Meikle, \& Allen 1990); for nearly a year there is no further 'revealing' of highly optically thick iron as the ejecta expands. Thus, there is little evidence from the near-IR forbidden lines for iron in the ejecta with $1 \ll \tau_{26 \mu m}<100$. While the evolution of the $26 \mu \mathrm{m}$ line intensity provides a challenge, together the temporal constancy of the optically thin near-IR [Fe II] line profiles, the sameness of the near-IR and mid-IR [Fe II] line profiles, and the ratio of the $18 \mu \mathrm{m}$ to the $26 \mu \mathrm{m}$ line profiles support the 'frothy iron finger' picture.

The velocity structure of Doppler-broadened profiles can arise through blending, optical depth, electron scattering from an expanding envelope, or asymmetry in the line- 
emitting region. Blending and optical depth effects are negligible for the $18 \mu \mathrm{m}$ and $1.26 \mu \mathrm{m}$ lines. Electron scattering shifts the line centroid redward and produces a strong asymmetric red wing (Fransson \& Chevalier 1989). Witteborn et al. (1989) model the profile of the [Ar II] $6.98 \mu \mathrm{m}$ line by spherically symmetric electron scattering from the low density $10 M_{\odot} \mathrm{H}$ envelope with an ionization fraction of $10 \%$, requiring an optical depth of $\tau_{e} \sim 0.4$ at 415 days. A more recent analysis by Xu et al. (1991) indicates that the hydrogen volume emission measure arises from a $1 \%$ ionization fraction high-density $1 M_{\odot} \mathrm{H}$ region near or within the metal-rich volume which produces a negligible electron scattering optical depth. We then are left to consider the asymmetric line profiles as arising from an asymmetric distribution of high-velocity fingers of iron-group elements (Spyromilio et al. 1990). The spatially inhomogeneous distribution of iron-group elements arises first by the Rayleigh-Taylor instabilities in the early phases of the explosion, as demonstrated by hydrodynamic simulations by Fryxell, Arnett, \& Muller 1991, Herant, Benz, \& Colgate 1992, and others. Secondly, the hot ${ }^{56}$ Co clumps or fingers do work on the surrounding gas, compressing the non-iron group elements and expanding to fill roughly half the metal-rich volume of the ejecta ( $\mathrm{Li}$ et al. 1993).

\section{Stable Nickel in the Ejecta}

Stable nickel is created along with radioactive nickel in explosive silicon burning and thereby is a trace constituent of the iron-group fingers in the ejecta. All forbidden lines of all ionic species of nickel arising from ground state terms with $E_{u} / k \leq 5000 \mathrm{~K}$ occur in the $2 \mu m-14.5 \mu m$ wavelength range, including [Ni I], [Ni II], [Ni III], and [Ni IV]. Spectra of the [Ni I] and [Ni II] forbidden lines were obtained from the KAO at 260, 415, 615, 775, and 1140 days (Wooden et al. 1993, Colgan et al. 1994, Witteborn et al. 1990). Forbidden lines of [Ni III] and [Ni IV] were not detected. Owing to the relatively low abundance of nickel compared to iron, the mid-IR forbidden lines of stable nickel suffer less optical depth effects than the mid-IR iron lines. The nickel lines are therefore ideal for studying the degree of ionization in the inner regions of the ejecta.

The only singly-ionized nickel forbidden line with $E_{u} / k<10000 K$ is the ground state [Ni II] $a^{2} D_{3 / 2}-a^{2} D_{5 / 2} 6.634 \mu m$ line with $E_{u} / k=2168 K$; this is the strongest mid-IR forbidden line in the spectrum of SN 1987A through 1140 days. The [Ni II] $6.6 \mu \mathrm{m}$ line has a critical density of $10^{7} \mathrm{~cm}^{-3}$ (Nussbaumer \& Storey 1988) and must be treated in non-LTE. The strongest mid-IR neutral nickel transition is the ground state [Ni I] $a^{3} F_{3}-a^{3} F_{4} 7.507 \mu m$ line with $E_{u} / k=1917 \mathrm{~K}$, although there are other [Ni I] forbidden lines at $3.12 \mu \mathrm{m}, 3.95 \mu \mathrm{m}, 5.89 \mu \mathrm{m}, 11.308 \mu \mathrm{m}$, and $12.00 \mu \mathrm{m}$. The [Ni I] $7.5 \mu \mathrm{m}$ line has a critical density of $\sim 10^{5} \mathrm{~cm}^{-3}$, derived using the estimated collision strengths by Hollenbach and McKee (1989) at $T_{e} \simeq 3000 \mathrm{~K}$ (Wooden et al. 1993). The [Ni I] $7.5 \mu \mathrm{m}$ and the [Ni II] $6.6 \mu \mathrm{m}$ lines originate from upper levels with nearly identical excitation energies. The ratio of these two lines, $I([N i I] 7.5 \mu \mathrm{m}) / I([N i I I] 6.6 \mu \mathrm{m})$, is independent of the electron temperature for $T_{e} \leq 1000 K$, weakly dependent on the electron density $n_{e}$, and highly sensitive to the ionization fraction $x_{N i}$.

Figure 3 shows the predicted line ratio $I([N i I] 7.5 \mu m) / I([N i I I] 6.6 \mu m)$ as a function of nickel ionization fraction $x_{N i}$ for LTE $\left(\sim n_{e} \geq 10^{8} \mathrm{~cm}^{-3}\right)$ and for electron densities $n_{e}=10^{7} \mathrm{~cm}^{-3}, 10^{6} \mathrm{~cm}^{-3}$, and $10^{4} \mathrm{~cm}^{-3}$ at $T_{e}=3000 \mathrm{~K}$. The strongest constraints for both the ionization fraction and the total nickel mass are determined by the measurements at 415 days when both the [Ni II] $6.6 \mu \mathrm{m}$ and the [Ni I] $7.5 \mu \mathrm{m}$ lines are optically thin. The statistically weighted averages of the low and medium resolution line strengths are $I(7.5 \mu \mathrm{m})=3.2 \pm 0.9 \times 10^{-18} \mathrm{~W} \mathrm{~cm}^{-2}$ and $I(6.6 \mu \mathrm{m})=$ $60.2 \pm 1.2 \times 10^{-18} \mathrm{~W} \mathrm{~cm}^{-2}$ at 415 days (Wooden et al. 1993). For a range of elec- 


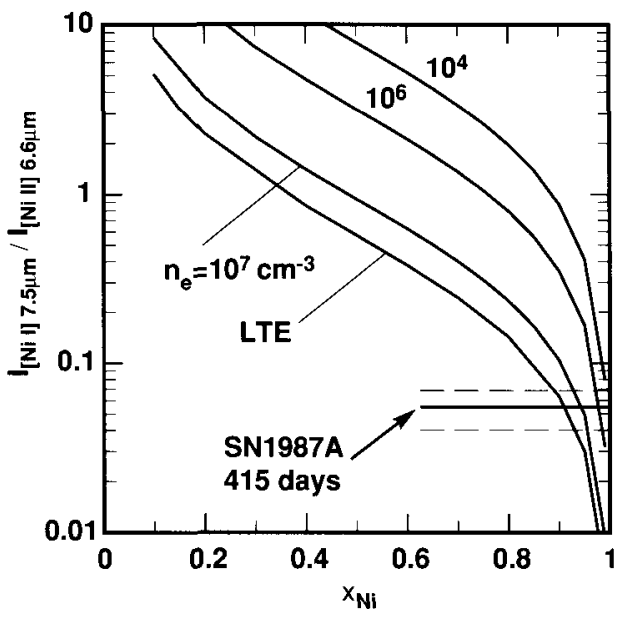

Figure 3. nickel ionization fraction

tron temperatures $T_{e}=3000-4500 K$ (Haas et al. $1990, \mathrm{Li}$, et al. 1993 ), the line intensity ratio $I\left(\left[\begin{array}{ll}N i & I\end{array}\right] 7.5 \mu \mathrm{m}\right) / I\left(\left[\begin{array}{ll}\mathrm{Ni} & \mathrm{II}\end{array}\right] 6.6 \mu \mathrm{m}\right)=0.05 \pm .01$ constrains the ionization fraction to be $x_{N i} \geq 0.90$. For LTE and $x_{N i}=0.90$, a total stable nickel mass of $M_{N i}=1.8-2.2 \times 10^{-3} M_{\odot}$ is derived for $T_{e}=4500-3000 K$. For a lower electron density of $n_{e}=5 \times 10^{6} \mathrm{~cm}^{-3}$ and $x_{N i}=0.95-0.97$, a higher mass of $M_{N i}=4.5-5.0 \times 10^{-3} M_{\odot}$ is derived (Wooden et al. 1993). The derived nickel ionization fraction of $x_{N i}(415$ days $) \geq 0.9$ is significantly higher than the $x_{N i}=0.6$ predicted by Li et al. (1993) for 415 days, but does agree with their predictions for times after 800 days. In $\mathrm{Li}$ et al. 's models, at about 600 days, the iron ionization fraction drops and the nickel ionization fraction increases to $x_{N i} \approx 1$ due to charge exchange between iron and nickel, but the charge exchange rates are not enumerated; perhaps these charge exchange effects are operable at 415 days? The derived nickel mass of $M_{N i}=4.5-5.0 \times 10^{-3} M_{\odot}$ is close to the predicted nickel mass from Woosley, Pinto, \& Weaver (1988)'s nucleosynthesis explosion models for an $18 M_{\odot}$ and a $20 M_{\odot}$ precursor but about 4 times smaller than the nickel mass predicted by Hashimoto, Nomoto, \& Shigeyama (1989)'s model for a $20 M_{\odot}$ precursor. The similarity of the shapes of the line profiles of the [Ni I] ${ }^{*} 3.12 \mu \mathrm{m}$, [Fe II] $1.26 \mu m$, [Fe II] $18 \mu m$, and the [Fe II] $26 \mu m$ lines (Spyromilio et al. 1990, Haas et al. 1990) indicates that the stable nickel and the iron occupy the same volume of the ejecta. The nickel has a relatively low abundance by number compared to the iron, so the iron provides the majority of electrons which collide with and excite the forbidden nickel lines.

\section{The Thermal Dust Continuum Emission}

A dramatic drop in the visual light output of the supernova commenced around 530 days (Whitelock et al. 1989): the $\mathrm{U}$ to $\mathrm{M}$ light curve declined more rapidly than expected for the radioactive decay of ${ }^{56} \mathrm{Co}$ and the ejecta's decreasing opacity to $\gamma$-rays. This change in the light curve at 530 days signifies the rapid formation of grains in the ejecta which is supported by the following three observables:

(a) At 615 days, the thermalized ${ }^{56} \mathrm{Co} \gamma$-ray luminosity $L_{56, \gamma}$ equals the sum of the UV, visual, and IR luminosities. The energy budget is balanced when the drop if the visual light is compensated by the increase in the thermal IR continuum (Suntzeff \& Bouchet 1990). 

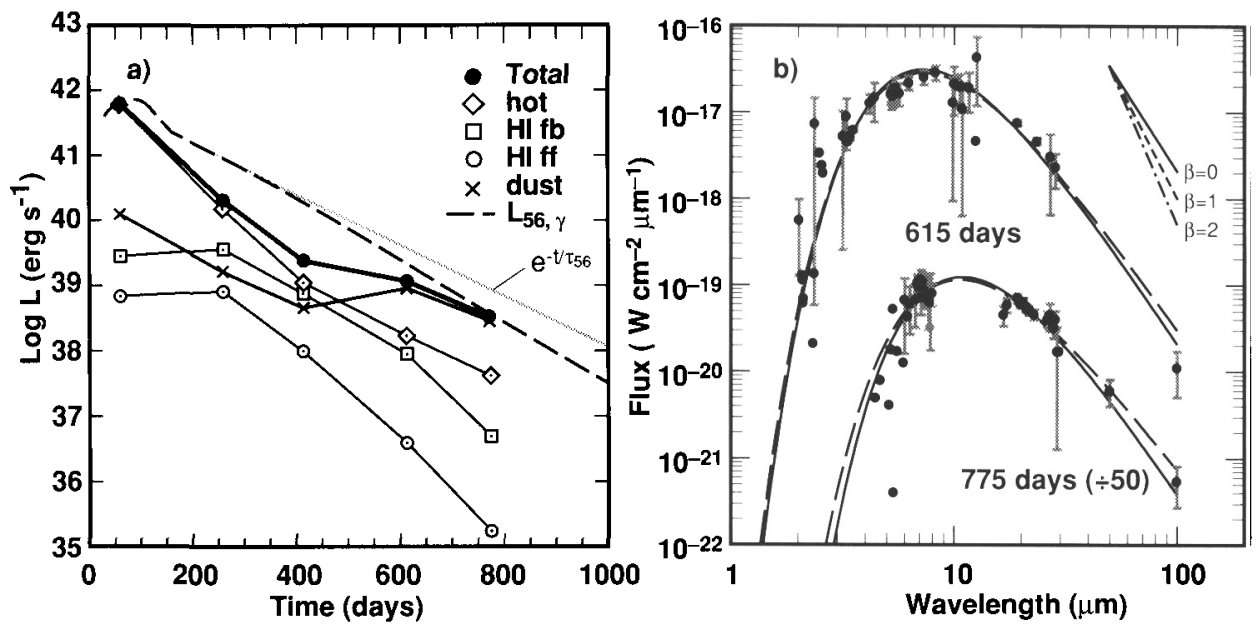

FIGURE 4. a. Temporal evolution of IR continuum luminosities. b. Dust continuum and $\chi^{2}$-fits.

(b) If $100 \%$ of the dust luminosity at 415 days is due to an IR echo off the circumstellar ring, then at most $14 \%$ of the observed dust luminosity at 615 days can be due to the ring; at 615 days $86 \%$ or more of the observed dust luminosity is due to newly condensed dust within the ejecta (Wooden et al. 1993).

(c) After $\sim 550$ days optical line profiles of $\mathrm{Mg} \mathrm{I}] 4571 \stackrel{\circ}{A}$, [O I] $6300 \AA$, [C I] $9844 \AA$ (Lucy et al. 1989a, 1989b), and infrared profiles [Si I], [Fe II], [Ni II] become increasingly blue shifted with time (Spyromilio et al. 1990, Colgan et al. 1994). As dust condenses interior to a line-emitting volume the emission from the far side of the ejecta suffers greater extinction compared to the near side and the line centroids become blue-shifted.

The thermal dust continuum from SN 1987A comprises one of three IR continuum components, where the HI free-free plus free-bound continuum emission and the RayleighJeans tail of a hot $\sim 5000 \mathrm{~K}$ blackbody continuum emission are the other two components. The best- $\chi^{2}$-fit IR continuum models yield the luminosity in each of the IR continuum components at 60, 260, 415, 615, and 775 days as shown in Figure 4a (Wooden et al. 1993). At early times $(60,260$, and 415 days), the dust luminosity is $2 \%-3 \%$ of the thermalized ${ }^{56} \mathrm{Co} \gamma$-ray luminosity $\left(L_{56, \gamma}-\right.$ Woosley, Pinto, \& Hartmann 1988$)$. At 615 days, the dust luminosity increases dramatically to $45 \%$ of $L_{56, \gamma}$. At 775 days, the dust luminosity is $83 \%$ of $L_{56, \gamma}$. At even later times, the dust luminosity is $\sim 90 \%$ of $L_{56+57, \gamma}$ (Bouchet - this volume).

From the KAO observations of the dust's thermal emission spectrum at 615 and 775 days, the grains have a gray emissivity out to $30 \mu m-100^{\circ} \mu m$ as shown in Figure $4 \mathrm{~b}$. This is unlike other dusty astronomical objects which show grains with emissivities which fall as $\lambda^{-\beta}$ for $\beta=1,2$ compared to a blackbody (Hildebrand 1983). Either the grains in the ejecta are very large with radii $a>6 \mu m$ or the optical depth of the IR emission is very high with $\tau_{d}(30 \mu \mathrm{m})>1$. An optical depth of $\tau_{d, I R}(30 \mu \mathrm{m}) \approx 1$ requires a dust mass of $3.1 \times 10^{-4} M_{\odot}$ at 615 days, and a dust mass of $5.0 \times 10^{-4} M_{\odot}$ at 775 days. (The dust masses are derived from the graybody $\beta=0$ models shown in Fig. $4 \mathrm{~b}$ with best-fit parameters $( \pm 3 \sigma)$ from Wooden et al. 1993: at 615 days $T_{d}=400_{-12}^{+12}, F_{d}(7.8 \mu m)=$ $3.08_{+0.40}^{-0.40} \times 10^{-17} \mathrm{~W} \mathrm{~cm}^{-2} \mu \mathrm{m}^{-1}$ and at 775 days $T_{d}=266_{-23}^{+23}, F_{d}(7.8 \mu \mathrm{m})=0.46_{+0.15}^{-0.15} \times$ $10^{-17} \mathrm{~W} \mathrm{~cm}^{-2} \mu \mathrm{m}^{-1}$.) The visual light which does escape appears to suffer a $\lambda^{-1}$ extinction law (Lucy et al. 1989a, 1989b). At 775 days, the dust mass required to produce the diffuse selective extinction by small amorphous carbon, graphite, or iron grains is an 
order of magnitude smaller than the minimum dust mass derived from the IR thermal continuum (Lucy et al. 1989b). A larger mass of small silicate grains comparable to the optically thick dust mass would be required to produce the diffuse selective extinction, but the distinct absence of a $9.7 \mu \mathrm{m}$ silicate emission feature in all mid-IR spectra and narrow band photometry of SN 1987A rules out silicate grains as the dominant grain component.

Taken together the dust's IR thermal continuum and the dust's visual extinction indicate that the grains in the ejecta of SN 1987A are small grains, with the bulk of the grains residing in optically thick regions (Lucy et al. 1989b, Wooden et al. 1993). In addition, the temporal evolution of the dust temperatures is consistent with the grains being immersed in an optically thick medium with a gas-to-dust ratio that is constant in time (Wooden et al. 1993). It is convenient to think of the grains in optically thick 'clumps', although the dust optical depth arises from enhanced grain column depths along the observer's line-of-sight. If the dust is at an expansion velocity of $v_{d}=1800 \mathrm{~km} \mathrm{~s}$ then $400 K$ dust fills $55 \%$ of the surface area at 615 days and $266 K$ dust fills $56 \%$ of the surface area at 775 days. The surface area filling factor stays constant as the volume of the ejecta expands by a factor of 2 . At 615 days, $45 \%$ of the thermalized ${ }^{56} \mathrm{Co}$ $\gamma$-ray luminosity is being reradiated as dust luminosity, requiring that $0.67 M_{\odot}$ of gas be participating in converting $\gamma$-rays to UV and visible photons which in turn heat the dust. At 775 days, $83 \%$ of the thermalized ${ }^{56}$ Co $\gamma$-ray luminosity is being reradiated as dust luminosity, more than doubling the mass of gas to $1.6 M_{\odot}$ participating in heating the dust (Wooden et al. 1993). The surface area filling factor remains constant while the mass of gas participating in heating the dust more than doubles. Together this suggests that dust forms interior to and along the same radial-lines-of-sight as the first dust formed.

There is evidence for the dust condensation within the iron-rich volume of the ejecta by consideration of the change in the iron-group element emission line profiles after about 575 days. While the $\mathrm{He}$ I $1.083 \mu \mathrm{m}$, Paschen $\beta 1.282 \mu \mathrm{m}$, and $\mathrm{Mg}$ I $1.503 \mu \mathrm{m}$ lines become blue-shifted with the same $\lambda^{-1}$ dependence as the optical Mg I] and [O I] lines, the irongroup lines ([Fe II] $1.257 \mu m$, [Fe II] $1.553 \mu \mathrm{m},[\mathrm{Fe} \mathrm{II}] 1.664 \mu \mathrm{m}$, and [Ni I] $3.119 \mu \mathrm{m}$ ) undergo a blueward shift about three times greater (Spyromilio et al. 1990). At 615 days and more so at 775 days the [Ni II] $6.634 \mu \mathrm{m}$ line also becomes blue-shifted (Colgan et al. 1994, Wooden et al. 1993). The $-440 \pm 270 \mathrm{~km} \mathrm{~s}^{-1}$ blue-shift seen in the 615 day high resolution measurements of the [Ni II] $6.634 \mu \mathrm{m}$ line is successfully modeled by placement of optically thick clumps of dust distributed proportional to the gas density interior to $v_{d} \simeq 1900 \mathrm{~km} \mathrm{~s}^{-1}$ (Colgan et al. 1994). The line intensities of the near-IR [Fe II] $0.716 \mu m, 0.862 \mu m$, and the $1.258 \mu m$ lines drop significantly between 575 and 735 days: the small mass of emitting iron at 735 days may be due to the iron being hidden in optically thick clumps of dust or the iron condensing into dust (Spyromilio \& Graham 1992). The condensation of small iron-rich grains, i.e., $\mathrm{Fe} / \mathrm{Ni}$ grains or FeS grains, in the ejecta of SN 1987A explains the observed $\lambda^{-1}$ extinction law, the absence of a $9.7 \mu \mathrm{m}$ silicate emission feature, and may, in the case of FeS grains (Pollack et al. 1994), provide the observed gray emissivity through far-IR wavelengths. The dusty 'clumps' could very well be the 'dusty frothy iron fingers'!

\section{Acknowledgements}

We acknowledge NASA's support of the Airborne Astronomy Program and the commitment of the staff and the crew of the Kuiper Airborne Observatory to the acquisition of this unique set of observations of SN 1987A. 


\section{REFERENCES}

Colgan, S. W. J., Haas, M. R., Erickson, E. F., Lord, S. D., \& Hollenbach, D. J. 1994, ApJ, 427,824

Dwek, E. 1988, Proc. ASA, 7, 468

Dwek, E., Moseley, S. H., Glaccum, W., Graham, J. R., Loewenstein, R. F., Silverberg, R. F., \& Smith, R. K. 1992, ApJ, 389, L21

Erickson, E. F., Haas, M. R., Colgan, S. E., Lord, S. D., Burton, M. G., Wolf, J., Hollenbach, D. J., \& Werner, M. 1988, ApJ, 330, L39

Fransson, C. \& Chevalier, R. A. 1989, ApJ, 343, 323

Fryxell, B., Miller, E., \& Arnett, D. 1991, ApJ, 367, 619

Haas, M. R., Colgan, S. W. J., Erickson, E. F., Lord, S. D., Burton, M. G., \& Hollenbach, D. J. 1990, ApJ, 360, 257

Harvey, P., Lester, D., Dinerstein, H., Smith, B., \& Colome, C. 1989, BAAS, 21, 1215

Hashimoto, M., Nomoto, K., \& Shigeyama, T. 1989, A\&A, 210, L5

Herant, M., Benz, W., \& Colgate, S. 1992, ApJ, 395, 642

Hildebrand, R. H. 1983, QJRAS, 24, 267

Hollenbach, D. \& McKee, C. F. 1989, ApJ, 342, 306

Li, H., McCray, R., \& Sunyaev, R. A. 1993, ApJ, 419, 824

Lucy, L. B., Danziger, I. J., Gouiffes, C., \& Bouchet, P. 1989a, in Proc. IAU. Coll. 120, Structure and Dynamics of the Interstellar Medium, ed. G. Tenorio-Tagle, M. Moles, \& J. Melnick (Berlin: Lecture Notes in Physics, Springer-Verlag), 164

Lucy, L. B., Danziger, I. J., Gouiffes, C., \& Bouchet, P. 1989b, in Supernovae: The Tenth Santa Cruz Workshop in Astronomy and Astrophysics, ed. S. E. Woosley (New York: SpringerVerlag), 82

Meikle, W. P. S., Allen, D. A., Spyromilio, J., \& Varani, G.-F. 1989, MNRAS, 238, 193

Moseley, S. H., Dwek, E., Glaccum, W., Graham, J. R., Loewenstein, R. F., \& Silverberg, R. F. 1989a, ApJ, 347, 1119

Moseley, S. H., Dwek, E., Glaccum, W., Graham, J. R., Loewenstein, R. F., \& Silverberg, R. F. 1989 b, Nature, 340,697

Nussbaumer, H. \& Storey, P. J. 1988, A\&A, 200, L25

Oliva, E., Moorwood, A. F. M., \& Danziger, I. J. 1987, ESO Messenger, No. 50, 18

Pollack, J. B., Hollenbach, D., Simonelli, D. P., Beckwith, S., Roush, T., \& Fong, W. 1994, ApJ, 421,615

Rank, D., Pinto, P. A., Woosley, S. E., Bregman, J. D., Witteborn, F., Axelrod, T. S., \& Cohen, M. 1988b, Nature, 331, 505

Spyromilio, J., Meikle, W. P. S., \& Allen, D. A. 1990, MNRAS, 242, 669

Spyromilio, J. \& Graham, J. R. 1992, MNRAS, 255, 671

Suntzeff, N. B. \& Bouchet, P. 1990, AJ, 99, 650

Whitelock, P. A. et al. 1989, MNRAS, 240, 7P

Witteborn, F. C., Bregman, J. D., Wooden, D. H., Pinto, P. A., Rank, D. M., Woosley, S. E., \& Cohen, M. 1989, ApJ, 338, L9

Witteborn, F. C. et al. 1990, BAAS, 22,1147

Woosley, S. E., Pinto, P. A., \& Weaver, T. A. 1988, Proc. ASA, 7, 355

Woosley, S. E., Pinto, P. A., \& Hartmann, D. 1989, ApJ, 346, 395

Wooden, D. H., Rank, D. M., Bregman, J. D., Witteborn, F. C., Tielens, A. G. G. M., Cohen, M., Pinto, P. A., Axelrod, T. S. 1993, ApJS, 88, 477

Xu, Y., McCray, R., Oliva, E., \& Randich, S. 1991, ApJ, 386, 181 